\title{
Heat and Mass Transfer Model for Droplets with Internal Circulation
}

\author{
Mathias Poulsen Kim Sørensen Thomas Condra \\ Department of Energy Technology, Aalborg University, Denmark, \{mps, kso, tc $\}$ @et .aau.dk
}

\begin{abstract}
In large droplets the internal resistance to heat or mass transfer has to be accounted for. Two respective models for low and high Reynolds numbers are investigated. The models are solved numerically for a range of modified Peclet numbers and the corresponding transfer numbers, representing either the Nusselt or Sherwood numbers, are determined. The results for each model are fitted to produce an expression that can be easily evaluated for use in a CFD code. The fits has mean deviations of $0.63 \%$ and $0.035 \%$ for the low and high Reynolds number models respectively. A proposed switching Reynolds number is used to combine the models and the combined model is compared to temperature measurements of free falling water droplets. It was found that the model is in good agreement with the data for the smallest droplets whereas it deviates as much as $40 \%$ for the larger droplets in the data set.
\end{abstract}

Keywords: internal circulation, mass transfer, heat transfer, droplets, CFD

\section{Introduction}

The droplet diameter in sprays with a large mass flow are typically in the order of $10^{-3}-10^{-5} \mathrm{~m}$. When modelling heat and mass transfer for small droplets, the transfer coefficients inside the droplets can be assumed to be infinite, and the overall transfer coefficients can be determined externally, by utilising correlations such as the FrösslingMarshall equation (Bird et al., 2002). However, in larger droplets the heat and mass transfer coefficient is significantly lower and this must be taken into consideration.

Processes involving sprays are often complex two-phase flow with many different types of interactions between the gas and liquid. Computational fluid dynamics (CFD) is an exceptional tool for capturing the respective gas-liquid interactions. As CFD requires relatively large computational capacity, the models used to describe the gas-liquid interaction should be fairly simple to evaluate, so as to limit the computational resources necessary.

\section{Governing Equations}

When modelling a two phase flow, the transfer of heat and mass are expressed as functions of intrinsic phase properties, that being temperature or mass concentration. The flux across the droplet interface can be described from both the gas and droplet side as:

$$
\begin{aligned}
& j=\kappa_{g}\left(\phi_{g}-\phi_{g}^{i f}\right)(\text { gas side }) \\
& j=\kappa_{d}\left(\phi_{d}^{i f}-\phi_{d}\right) \text { (droplet side) }
\end{aligned}
$$

where $j$ is the flux across the interface, $\phi$ is the driving potential, e.g. mass concentration or temperature of the phase on the gas or droplet side, $\phi^{i f}$ is the potential at the interface and $\kappa$ is the transfer coefficient on each side of the interface.

For larger droplets the internal movement of liquid causes a non-uniform distribution of the transfer quantity, which in turn causes the transfer coefficient to vary depending on the distribution of mass or heat. To describe the heat or mass transport inside the droplet a continuity equation can be expressed for the quantity of interest as:

$$
\frac{\partial \phi}{\partial t}+\nabla u \phi=\nabla(\Gamma \nabla \phi)
$$

where $u$ is the internal droplet velocity, and $\Gamma$ is the mass or thermal diffusion coefficient.

In the analysis below, the following assumptions are made:

\section{A. 1 The droplet is spherical}

A. 2 The fluid of the droplet is incompressible and viscous

A. 3 Fluid properties are constant throughout the droplet and independent of $\phi$

A. 4 The distribution of $\phi$ and velocity field is axisymmetric

A. 5 The velocity field time independent

A. 6 The droplet interface is at equilibrium with the gas phase

\subsection{Low Reynolds Model}

At low Reynolds numbers the streamlines described by (Hadamard, 1911) can be used to calculate the velocity field within the droplet. The streamlines are shown in the right half of Figure 1. The streamline function in spherical coordinates can be given as:

$$
\psi(r, \theta)=\frac{1}{4} u_{r e l} a^{2} \sin ^{2}(\theta)\left(\frac{\mu_{g}}{\mu_{g}+\mu_{d}}\right)\left(\frac{r}{a}\right)^{2}\left[1-\left(\frac{r}{a}\right)^{2}\right]
$$


where $r$ and $\theta$ are the coordinates with origin at the centre of the droplet, $u_{r e l}$ is the relative velocity between the droplet and gas, $\mu$ is the dynamic viscosity of the gas and droplet respectively and $a$ is the radius of the droplet.

The respective velocity components can be derived from the stream function in Equation 4 by (Munson et al., 2013):

$$
\begin{aligned}
u_{r} & =\frac{1}{r^{2} \sin (\theta)} \frac{d \psi(r, \theta)}{d \theta} \\
u_{\theta} & =-\frac{1}{r \sin (\theta)} \frac{d \psi(r, \theta)}{d r}
\end{aligned}
$$

This approach has previously been investigated by Wellek et al. (1970), where the continuity equation presented in Equation 3 is rearranged to make it dimensionless.

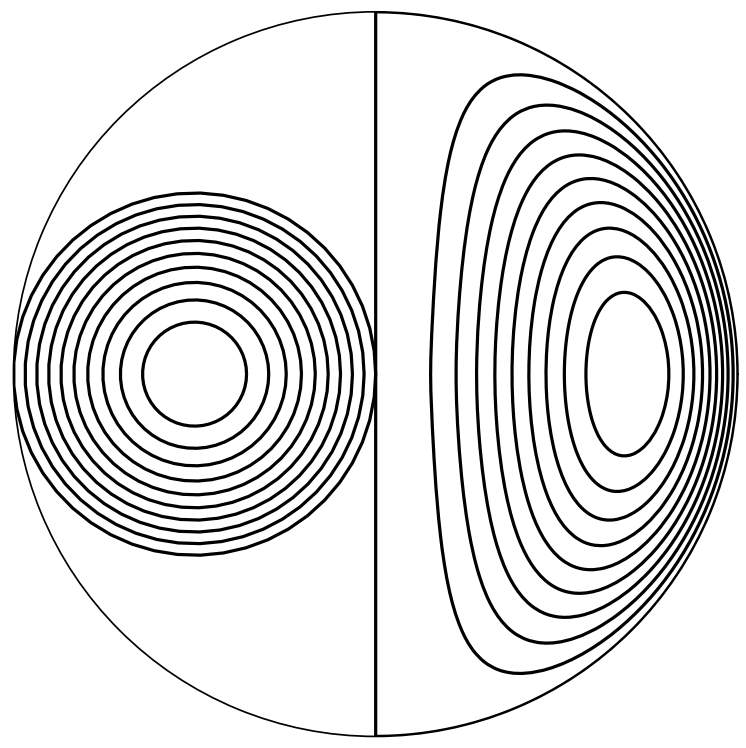

Figure 1. Streamlines inside the droplet. On the left side is the tori streamlines for the high Reynolds model and on the right side is the Hadamard streamlines for the low Reynolds model.

The dimensionless transport equation in spherical coor- dinates is given as:

$$
\begin{gathered}
\frac{\partial \Phi}{\partial \tau}+\frac{1}{4}\left[P e^{\prime}\left(1-R^{2}\right) \cos (\theta)-\frac{2}{R}\right] \frac{\partial \Phi}{\partial R}-\frac{\partial^{2} \Phi}{\partial R^{2}} \\
+\frac{1}{4}\left[P e^{\prime} \frac{\left(2 R^{2}-1\right)}{R}-\frac{\cot (\theta)}{R^{2}}\right] \frac{\partial \Phi}{\partial \theta}-\frac{1}{R^{2}} \frac{\partial^{2} \Phi}{\partial \theta^{2}}=0 \\
\tau=\frac{t \Gamma}{(2 a)^{2}} \\
P e^{\prime}=\left(\frac{\mu_{g}}{\mu_{g}+\mu_{d}}\right) P e \\
P e=\frac{u_{r e l}(2 a)}{\Gamma} \\
R=\frac{r}{a} \\
\Phi=\frac{\phi(t)-\phi(0)}{\phi(\infty)-\phi(0)}
\end{gathered}
$$

Where $\tau$ is the Fourier number, $P e^{\prime}$ is the modified Peclet number, $R$ is the relative radial position and $\Phi$ is the normalised mass or heat.

By making Equation 3 dimensionless a solution for a given $P e^{\prime}$ can be used independent of the specie or liquid in question.

For the limiting cases where the transfer is dominated by either diffusion or convection i.e. $P e=0$ or $P e=\infty$, there are analytical solutions. These are expressed as volume averages of the quantity of interest over the droplet, which can be given as:

$$
\bar{\Phi}=\int_{V} \Phi d V
$$

For the case of pure diffusion the solution of Equation 7 can be given as (Newman, 1931):

$$
\bar{\Phi}=1-\frac{6}{\pi^{2}} \sum_{n=1}^{\infty} \frac{1}{n^{2}} \exp \left(-n^{2} \pi^{2} \tau\right)
$$

where $\bar{\Phi}$ is the volume averaged quantity of interest and $V$ is the droplet volume.

For the other case where the transfer is dominated by convection a solution was found by separation of variables (Kronig and Brink, 1951). This method results is an infinite sum where each addition requires a new coefficient and an eigenvalue. In the original work only one set of coefficients and eigenvalues was found. Later the number of coefficients and eigenvalues where extended (Oliver and Souccar, 2006). The solution can be given as:

$$
\bar{\Phi}=1-\frac{3}{8} \sum_{n=1}^{\infty} A_{n}^{2} \exp \left(-16 \lambda_{n} \tau\right)
$$

where $A_{n}$ and $\lambda_{n}$ is the respective coefficient and eigenvalue to the $\mathrm{n}^{\text {th }}$ addition to the sum. 


\subsection{High Reynolds model}

For a droplet at high Reynolds number a model based on the streamlines being replaced by a system of tori is used (Handlos and Baron, 1957), see the left half of Figure 1. In this model the transfer inside the droplet is based on eddy diffusion, which is expressed as function of the relative radial position inside the tori system. As only the tori are responsible for the transfer mechanism, the volume that contributes to transfer is $59 \%$ of the entire droplet.

The transfer equation for the high Reynolds model are given as:

$$
\frac{\partial \Phi}{\partial \tau}=\frac{P e^{\prime}}{128} \frac{1}{\zeta} \frac{\partial}{\partial \zeta}\left(\left(6 \zeta^{3}-8 \zeta^{2}+3 \zeta\right) \frac{\partial \Phi}{\partial \zeta}\right)
$$

where $\zeta$ is the coordinate centred at the tori.

A solution for the high Reynolds model was given by (Olander, 1966), but this is only valid for $P e^{\prime} \tau / 128>0.1$. The solution presented by (Olander, 1966) is given as:

$$
\bar{\Phi}=1-0.64 \exp \left(-\frac{2.80 P e^{\prime} \tau}{128}\right)
$$

With the limitation of Equation 18, it will not be used directly further analysis, but it will still be used to validate the implementation of the model.

\subsection{Boundary Conditions}

For the boundary at the surface of the droplet, assumption A. 6 gives the following condition:

$$
\Phi(1, \tau)=1
$$

For the low Reynolds model the boundary condition at the droplet halves can be given as:

$$
\frac{\partial \Phi(R, 0, \tau)}{\partial \theta}=\frac{\partial \Phi(R, \pi, \tau)}{\partial \theta}=0
$$

\subsection{Transfer coefficient}

The classical transfer models for heat or mass are typically correlated to each of their respective dimensionless group which can be expressed as:

$$
N_{t}=\frac{\kappa_{d}(2 a)}{\Gamma}
$$

where $N_{t}$ is the Sherwood or Nusselt number.

To determine the dimensionless group for the quantity of interest, it has to be correlated to $\bar{\Phi}$. By defining the dimensionless flux across the droplet interface and rewriting Equations 2 and 21 to express the transfer coefficient and an expression for $N_{t}$ can be found (Andoe, 1968):

$$
\begin{gathered}
J=\frac{2}{3} \frac{d \bar{\Phi}}{d \tau} \\
\kappa_{d}=\frac{j}{\phi_{p, d}^{i f}-\phi_{p, d}}=\frac{J}{1-\bar{\Phi}} \frac{2 a}{\Gamma} \\
N_{t}=\frac{J}{1-\bar{\Phi}}
\end{gathered}
$$

Following the method presented in (Kronig and Brink, 1951) and (Handlos and Baron, 1957), $N_{t}$ is evaluated for a given $P e^{\prime}$, when $\bar{\Phi}=1-e^{-1} \approx 0.632$

\section{Computational Methodology}

Due to the difference in the nature of the governing equations, each model is solved using a different method. For the low Reynolds model the solver scalarTransportFoam from the open source package OpenFOAM is used to solve Equation 7 (Weller et al., 1998). OpenFOAM is written for Cartesian coordinates, therefore Equation 7 is rewritten into the form of Equation 3. Here the velocity components are given as:

$$
\begin{aligned}
& u_{x}=u_{r} \cos (\theta)-R u_{\theta} \sin (\theta) \\
& u_{y}=u_{r} \sin (\theta)+R u_{\theta} \cos (\theta)
\end{aligned}
$$

The discretisation scheme used for the divergence terms is second order upwind, and the time scheme is CrankNicholson.

A structured axis symmetric mesh is created for the droplet half where the centre axis of the droplet is the symmetry axis. Three different meshes with increasing mesh densities of 2400, 5400 and 9600 cells are investigated for varying $P e^{\prime}$ to ensure that the solutions is independent of the mesh density. The time step for the different meshes where handled by defining a maximum allowable Courant number of 0.5. In Figure 2 the results for three different mesh densities are shown. It can be seen that there is no significant difference between solution on the different meshes. With a relative difference below $0.1 \%$ between
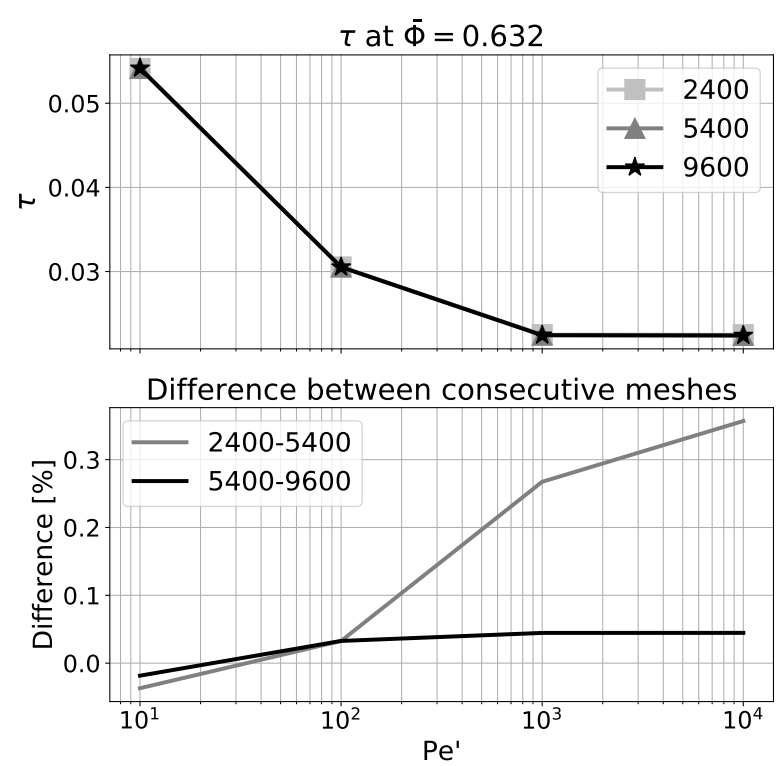

Figure 2. The top plot shows the dimensionless time for the three different mesh densities at different modified Peclet numbers. The bottom plot shows the relative difference between the consecutive meshes. 
the mesh with 5400 and 9600 cells, the mesh with 5400 cells are used for further analysis.

For the high Reynold model, Equation 16 is discretised using a second order accurate central finite difference approach. The first and second derivatives are expressed as:

$$
\begin{gathered}
\frac{\partial \Phi}{\partial \zeta} \approx \frac{\Phi_{+1}-\Phi_{-1}}{\Delta \zeta} \\
\frac{\partial^{2} \Phi}{\partial \zeta^{2}} \approx \frac{\Phi_{+1}-2 \Phi+\Phi_{-1}}{\Delta \zeta^{2}}
\end{gathered}
$$

For the dimensionless time a forward Euler approximation is used.

Just as for the low Reynolds model, an independence test was done to ensure that the solution is independent of the discretisation. In Figure 3 the value of $\tau$ when $\bar{\Phi}=$ 0.632 is shown for different values of the modified Peclet number $P e^{\prime}$.

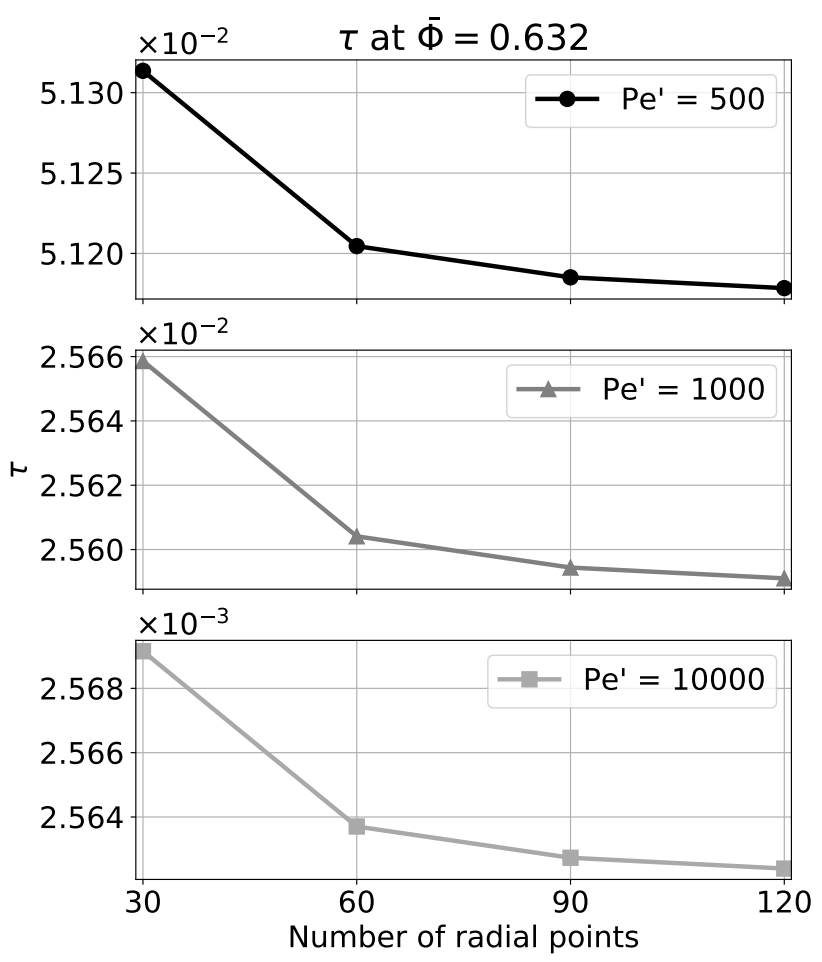

Figure 3. Independence test for the high Reynold model. The value of the dimensionless time $\tau$ when $\bar{\Phi}=0.632$ is shown at varying modified Peclet numbers for different number of radial points used to discretise the droplet.

With a relative difference of $0.013 \%$ or less, between the solution with 120 and 90 radial point for the investigated $P e^{\prime}$, the discretisation with 90 radial points are used for further analysis. A study of the dimensionless time step was also done, and it was found that a value of $1 \times 10^{-6}$ was adequate for all the modified Peclet numbers.

\subsection{Model Comparison}

As a final validation of the computational methodology, the numerical results from both models are compared with Equations 14, 15 and 18. The solution for the low Reynolds model at $P e^{\prime}=\infty$ is approximated by setting $P e^{\prime}=10^{4}$. The comparison for the high Reynolds model are shown for $P e^{\prime}=500$.

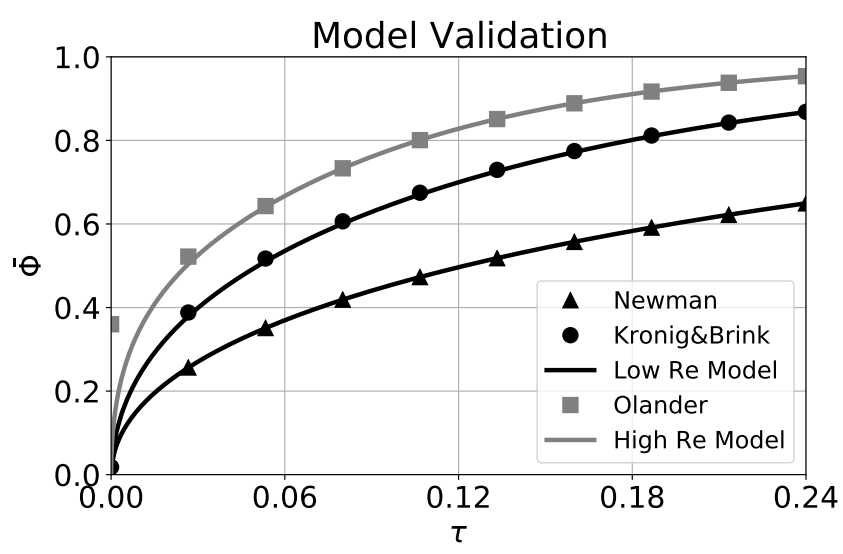

Low Reynolds Model High Reynolds Model

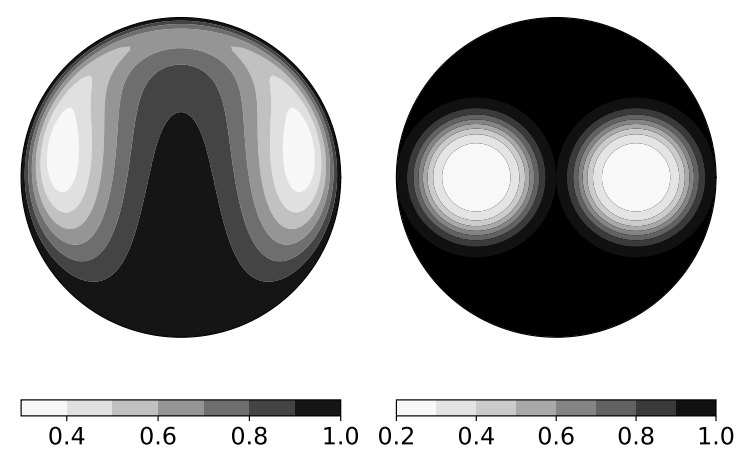

Figure 4. Comparison of the low and high Reynolds model with known solutions to their respective set of equations. On the bottom of the figure, the contours for the low and high Reynolds model are shown for $\bar{\Phi}=0.632$.

The output from the low Reynolds model at $P e^{\prime}=0$ and $P e=\infty$ agrees well with solution of Equations 14 and 15 , respectively. As stated by (Olander, 1966) the expression in Equation 18 is only accurate for $P e^{\prime} \tau / 128>0.1$, which is why the first point of comparison deviates from the model results, whilst for the remaining points it agrees with model.

\section{Results}

The models for low and high Reynold number are run over a span of modified Peclet numbers ranging from 0 to $10^{4}$ and the transfer number is evaluated for each modified Peclet number. The resulting transfer numbers are shown in Figure 5:

To get an expression for the transfer numbers as a function of $P e^{\prime}$ the two curves shown in Figure 5 are fitted using least-squares. The curve for the low Reynolds model transfer numbers resembles that of a step response to an under-damped second-order system shifted by an amount equal to that of the transfer number of the solution presented by (Newman, 1931), when $P e^{\prime} \leq 1000$. 


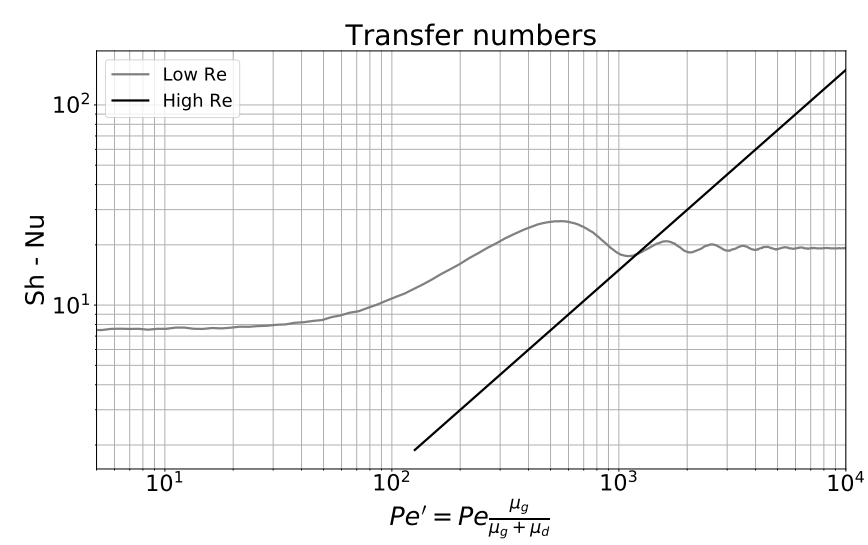

Figure 5. Resulting transfer numbers for the low and high Reynold model at varying modified Peclet numbers.

For $P e^{\prime}>1000$ it resembles that of a damped oscillation with a mean value equal to that of the transfer number for the solution presented by (Kronig and Brink, 1951). The transfer numbers for the solutions presented by (Newman, 1931) and (Kronig and Brink, 1951) are given as:

$$
\begin{aligned}
& N_{t, n m}=7.551 \\
& N_{t, k b}=19.18
\end{aligned}
$$

With these remarks in mind the following equations can represent the transfer numbers for the low Reynolds model:

For $P e^{\prime} \leq 1000$ :

$$
N_{t, l o w}=a\left[1-b \cdot \exp \left(-c \cdot P e^{\prime}\right) \sin \left(d \cdot P e^{\prime}+e\right)\right]+N_{t, n m}
$$

For $P e^{\prime}>1000$ :

$$
N_{t, l o w}=b \cdot \exp \left(-c \cdot P e^{\prime}\right) \sin \left(d \cdot P e^{\prime}-e\right)+N_{t, k b}
$$

The coefficients for Equations 31 and 32 are presented in Table 1 below:

Table 1. Coefficients for the low Reynolds model fit.

\begin{tabular}{lccccc}
\hline Model & $a$ & $b$ & $c$ & $d$ & $e$ \\
\hline Low & 1.249 & 1.030 & 1.269 & 5.169 & 1.677 \\
& $\times 10^{1}$ & $\times 10^{0}$ & $\times 10^{-3}$ & $\times 10^{-3}$ & $\times 10^{0}$ \\
High & - & 3.013 & 5.149 & 6.508 & 2.575 \\
& & $\times 10^{0}$ & $\times 10^{-4}$ & $\times 10^{-3}$ & $\times 10^{0}$ \\
\hline
\end{tabular}

The maximum error between the fit for the low Reynold model and numerical results is $3.8 \%$ and the mean error is $0.63 \%$, which are acceptable considering numerical uncertainties.

The fit for the high Reynolds model is simply a linear equation, which is given as:

$$
N_{t, h i g h}=1.523 \times 10^{-2} \cdot P e^{\prime}
$$

The maximum and mean errors for the high Reynold model are $0.035 \%$ and $0.025 \%$, respectively.

With the fits for the two models made these are combined into a single expression for the transfer number. It is proposed that the switching between the two models are determined by a switching Reynolds number, such that:

$$
N_{t}=\operatorname{neg}\left(R e-R e_{s w}\right) N_{t, l o w}+\operatorname{pos} 0\left(R e-R e_{s w}\right) N_{t, h i g h}
$$

where $R e$ is the external Reynolds number and $R e_{s w}$ is the switching Reynolds number.

The function $\operatorname{neg}(x)=1$ for all negative values and $\operatorname{neg}(x)=0$ for all positive. The function pos 0 is the opposite and includes 0 such that $\operatorname{pos} 0(0)=1$.

\section{Validation}

For the model validation the experimental data from (Yao and Schrock, 1976) is used. In this study water droplets with diameters of 3, 4,5 and $6 \mathrm{~mm}$ are investigated. The average temperature of the droplets are sampled at varying points throughout a free fall of $3 \mathrm{~m}$. Yao and Schrock (1976), made three experiments per droplet diameter at varying relative humidity of the surrounding air.

The experimental setup is modelled in an EulerEuler framework, using the OpenFOAM solver reactingTwoPhaseEulerFoam (Rusche, 2003). The governing equations for the two-phase system are given as:

$$
\begin{gathered}
\frac{\partial}{\partial t}\left(\alpha_{i} \rho_{i} u_{i}\right)+\nabla \cdot\left(\alpha_{i} \rho_{i} u_{i}\right)=\sum_{j} \dot{m}_{i j} \\
\frac{\partial}{\partial t}\left(\alpha_{i} \rho_{i} u_{i}\right)+\nabla \cdot\left(\alpha_{i} \rho_{i} u_{i}\right)=-\alpha_{i} \nabla p+\nabla\left(\alpha_{i} \mu_{i} \nabla u_{i}\right)+\sum_{j} \vec{F}_{i j}
\end{gathered}
$$

where $\alpha_{i}$ is the volume faction of the ith phase, $\rho_{i}$ is the density of the ith phase, $u_{i}$ is the ith phase velocity, $\sum_{j} \dot{m}$ is the mass transfer between the phases, $p$ is the common pressure, and $\vec{F}_{i j}$ is the volumetric forces acting on the ith phase.

The forces considered to match the experimental contritions are gravity and drag. Where the drag coefficient for the liquid droplets are given as (Holterman, 2003):

$$
C_{d}=\left[\left(\frac{24}{R e}\right)^{0.52}+0.32^{0.52}\right]^{1 / 0.52}
$$

To account the effect of the relative humidity of the surrounding air, the temperature and mass concentration at the interface between the droplet and air has to be modelled. The temperature at the interface can be calculated by combining Equations 1 and 2 and adding the heat of evaporation. To solve this equation Newton acceleration is used. The initial guess is calculated without mass transfer. The interfacial temperature can be updated as follows:

$$
T^{i f}=T^{i f}-\frac{\kappa_{g}\left(T^{i f}-T_{g}\right)+\kappa_{g}\left(T^{i f}-T_{d}\right)+\dot{m}}{\kappa_{g}+\kappa_{d}+\frac{d \dot{m}}{d T}}
$$


The mass transfer in the form of evaporation or condensation at the interface are determined by the saturation pressure of water vapour, which is given by the Arden Buck equation:

$$
\begin{aligned}
& p_{\text {sat }}\left(T_{c}\right)= \\
& 611.21 \exp \left(\left(18.678-\frac{T_{c}}{234.5}\right)\left(\frac{T_{c}}{257.14+T_{c}}\right)\right)
\end{aligned}
$$

where $p_{\text {sat }}$ is the saturation pressure given in $\mathrm{Pa}$ and $T_{c}$ is the temperature in degrees Celsius.

The Nusselts correlation for the external side of the droplet are given as (Ahmed and M.Yovanovich, 1994)

$$
\begin{gathered}
N u=2+0.775 \operatorname{Re}^{0.5} \frac{\operatorname{Pr}^{1 / 3} / \sqrt{2 \gamma+1}}{\left[1+\left(\frac{1}{(2 \gamma+1)^{3} P r}\right)\right]^{1 / 6}} \\
\gamma=\frac{1}{(1+R e)^{0.25}}
\end{gathered}
$$

The simulation is 2-D, were a small volume at the top of the domain is prescribed a volumetric fraction of water of 0.005 with the temperature reported by (Yao and Schrock, 1976). The small volume of water and the computational mesh is shown below:

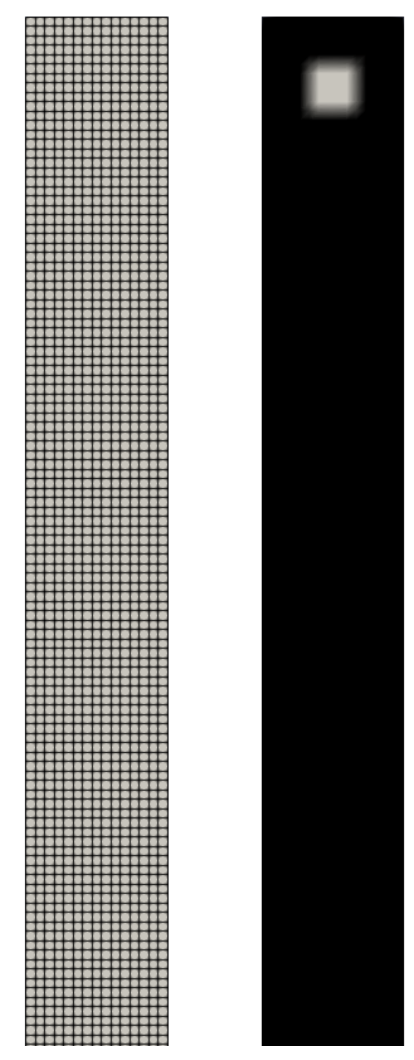

Figure 6. Computational mesh and the droplets at rest in the volume at the top of the domain.

In the simulation the sides are given a symmetric boundary condition, to avoid any wall effects. The total length of the domain is $4 \mathrm{~m}$ to ensure that the boundary at the bottom does not interfere with the results. The temperature of the air is kept at a constant temperature, corresponding to that reported for the individual experiments.

It was found the switching Reynolds number $R e_{s w}$ proposed in equation 34 should be 400 , to produce the best match with the experimental results.

The results of the simulations are compared with the experimental results, and are shown in Figure 7:

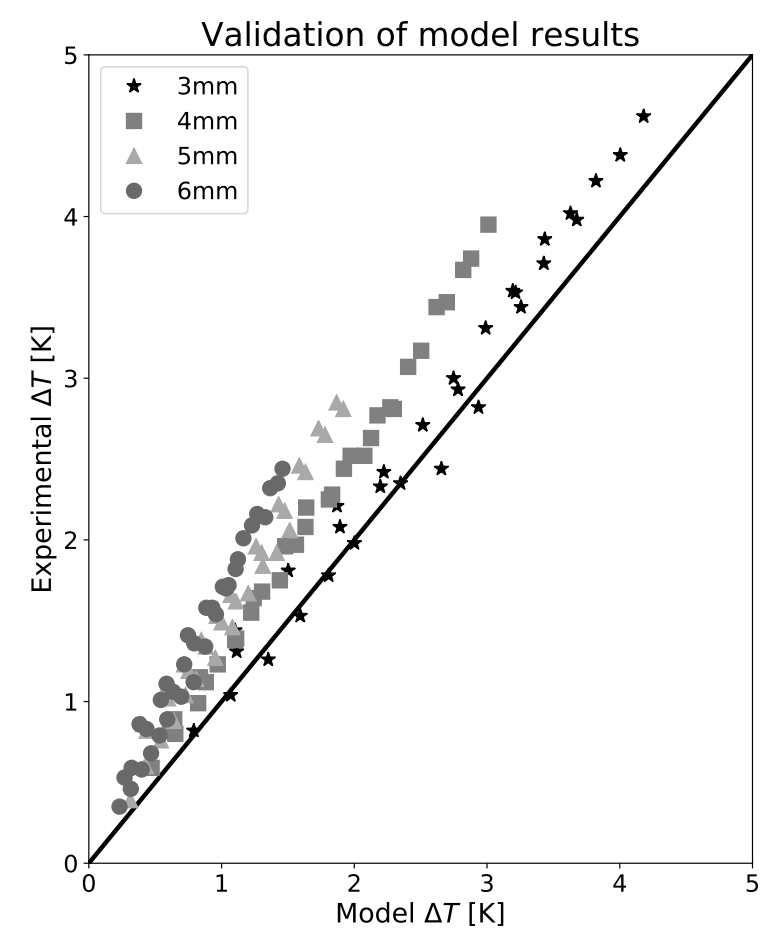

Figure 7. Simulations results compared with the experimental results of (Yao and Schrock, 1976). The results are presented as temperature decrease of the water droplets as it falls from rest.

It can be seen from Figure 7 that the simulation under predicts the decrease in water temperature as the diameter of the droplets increases. In the worst cases the results deviate by $40 \%$ compared to the experimental data.

\section{Conclusion}

Two models for large droplets with internal circulation have been investigated and an expression for the transfer number, Nusselt or Sherwood, has been found as a function of the modified Peclet number. The equations describing the fit of the numerical results were found to be within a satisfactory margin of error compared to the numerical results with a maximum error of $3.8 \%$. A switching Reynolds number was proposed as a method for combining the two models.

The model for the droplets was implemented in a twophase simulation, and compared to experimental data for 
droplets experiencing free fall. The results of the simulations were compared with the experimental data and it was found that it under predicts the temperature decrease of the falling droplets. As the diameter of the droplet increases so does the error between the simulation and experimental data. In the worst case the simulated results deviates as much as $40 \%$.

\section{References}

G. Ahmed and M.Yovanovich. Approximate analytical solution of forced convection heat transfer from isothermal spheres for all prandtl numbers. Journal of Heat Transfertransactions of The Asme - J HEAT TRANSFER, 1994. doi:10.1115/1.2911456.

William Victor Andoe. Liquid extraction with single dropletseffect of chemical reaction. $\mathrm{PhD}$ thesis, Missouri University of Science and Technology, 1968.

R. Byron Bird, Warren E. Stewart, and Edwin N. Lightfoot. Transport Phenomena. John Wiley \& Sons, Inc, 2nd edition, 2002. ISBN 0-471-41077-2.

J.S. Hadamard. Mouvement permanent lent d'une sphere liquide et visqueuse dans un liquide visqueux. C. R. Acad. Sci., 152: 1735-1752, 1911.

A.E. Handlos and T. Baron. Mass and heat transfer from drops in liquid-liquid extraction. AIChE, 3:127-136, 1957. doi:10.1002/aic.690030121.

H.J. Holterman. Kinetics and evaporation of water drops in air. CIP-DATA ROYAL LIBRARY, DEN HAAG, NL, 2003. ISBN 90-5406-234-7.

R. Kronig and J.C. Brink. On the theory of extraction from falling droplets. Applied Scientific Research, (2), 1951. doi:10.1007/BF00411978.

Bruce R. Munson, Ted H. Okiishi, Wade W. Huebsch, and Alric P. Rothmayer. Fluid Mechanics. John Wiley \& Sons, 7th edition, 2013. ISBN 978-1-118-318676.

Albert Broadus Newman. The drying of porous solid: Diffusions and surface emission equations. AIchE, 27:203-220, 1931.

Donald R. Olander. The handlos-baron drop extraction model. AIChE Journal, 12(5):1018-1019, 1966. doi:10.1002/aic.690120533.

Douglas L. Oliver and Adham W. Souccar. Heat transfer from a translating droplet at high peclet numbers: Revisiting the classic solution of kronig \& brink. Journal of Heat Transfer, 128(7):648-652, 2006. doi:10.1115/1.2193542.

Henrik Rusche. Computational fluid dynamics of dispersed twophase flows at high phase fractions. $\mathrm{PhD}$ thesis, Imperial College London, January 2003.

R. M. Wellek, W. V. Andoe, and R. J. Brunson. Mass transfer with chemical reaction inside single droplets and gas bubbles: Mathematical mechanisms. Can. J. Chem. Eng, 48(6):645655, 1970. doi:10.1002/cjce.5450480601.
H. G. Weller, G. Tabor, H. Jasak, and C. Fureby. A tensorial approach to computational continuum mechanics using objectoriented techniques. Computers in Physics, 12(6):620, 1998. doi:10.1063/1.168744.

Shi-Chune Yao and V. E. Schrock. Heat and mass transfer from freely falling drops. Journal of Heat Transfer, 98(1):120-126, February 1976. ISSN 0022-1481. doi: $10.1115 / 1.3450453$.

\section{Nomenclature}

\begin{tabular}{|c|c|c|}
\hline Symbol & Description & Unit \\
\hline$a$ & Droplet radius & $\mathrm{m}$ \\
\hline$A_{n}$ & Coefficient for Equation 15 & - \\
\hline$C_{d}$ & Drag coefficient & - \\
\hline$\vec{F}$ & Volumetric force & $\mathrm{N} \mathrm{m}^{-3}$ \\
\hline$j$ & Heat or mass flux & $\begin{array}{l}\mathrm{J} \mathrm{m}^{-2} \mathrm{~s}^{-1} \\
\mathrm{~kg} \mathrm{~m}^{-2} \mathrm{~s}^{-1}\end{array}$ \\
\hline$J$ & Dimensionless surface flux & - \\
\hline$\dot{m}$ & Mass flow & $\mathrm{kg} \mathrm{s}^{-1}$ \\
\hline$N_{t}$ & Transfer number & - \\
\hline$N u$ & Nusselts number & - \\
\hline$p$ & Pressure & $\mathrm{Pa}$ \\
\hline $\mathrm{Pe}$ & Peclet number & - \\
\hline$P e^{\prime}$ & Modified Peclet number & - \\
\hline $\operatorname{Pr}$ & Prandlt number & - \\
\hline$r$ & Radial coordinate & $\mathrm{m}$ \\
\hline$R$ & $\begin{array}{l}\text { Dimensionless } \\
\text { radial coordinate }\end{array}$ & - \\
\hline $\operatorname{Re}$ & Reynold number & - \\
\hline Sh & Sherwood Number & - \\
\hline$t$ & Time & $\mathrm{s}$ \\
\hline$T$ & Temperature & $\mathrm{K}$ \\
\hline$u$ & Velocity & $\mathrm{m} \mathrm{s}^{-1}$ \\
\hline$V$ & Volume & $\mathrm{m}^{3}$ \\
\hline$\alpha$ & Volume fraction & - \\
\hline$\Gamma$ & $\begin{array}{l}\text { Thermal conductivity } \\
\text { or mass diffusivity }\end{array}$ & $\mathrm{m}^{2} \mathrm{~s}^{-1}$ \\
\hline$\zeta$ & $\begin{array}{l}\text { Dimensionless tori centred } \\
\text { radial coordinate }\end{array}$ & - \\
\hline$\theta$ & Angle & - \\
\hline$\kappa$ & $\begin{array}{l}\text { Heat or mass } \\
\text { transfer coefficient }\end{array}$ & $\begin{array}{c}\mathrm{W} \mathrm{m}^{-2} \mathrm{~K}^{-1} \\
\mathrm{~m} \mathrm{~s}^{-1}\end{array}$ \\
\hline$\lambda_{n}$ & Eigenvalue for Equation 15 & - \\
\hline$\mu$ & Viscosity & $\mathrm{Pa} \mathrm{s}^{-1}$ \\
\hline$\rho$ & Density & $\mathrm{kg} \mathrm{m}^{-3}$ \\
\hline$\tau$ & $\begin{array}{l}\text { Dimensionless time/ } \\
\text { Fourier number }\end{array}$ & - \\
\hline$\Phi$ & Dimensionless potential & - \\
\hline $\bar{\Phi}$ & $\begin{array}{l}\text { Volume averaged } \\
\text { dimensionless potential }\end{array}$ & - \\
\hline$\Psi$ & Streamline & $\mathrm{m}^{3} \mathrm{~s}^{-1}$ \\
\hline
\end{tabular}




\begin{tabular}{ll}
\hline Symbol & Description \\
\hline subscripts \\
$c$ & Celsius \\
$d$ & Droplet \\
$g$ & Gas \\
$h i g h$ & High Reynolds model \\
$i$ & Phase $\mathrm{i}$ \\
$j$ & Phase $\mathrm{j}$ \\
$l o w$ & Low Reynolds model \\
$n m$ & Newman solution \\
$k b$ & Kronig and Brink solution \\
$r e l$ & Relative \\
$r$ & Radial \\
$s w$ & Switching \\
$\theta$ & Tangential \\
+1 & Step forward \\
-1 & Step backwards \\
\multicolumn{2}{c}{ superscripts } \\
if & Interface \\
\multicolumn{2}{l}{ abbreviations } \\
CFD & Computational Fluid Dynamics \\
\hline
\end{tabular}

